\title{
A PLAUSIBLE (OVERLOOKED) SUPER-LUMINOUS SUPERNOVA IN THE SLOAN DIGITAL SKY SURVEY STRIPE 82 DATA
}

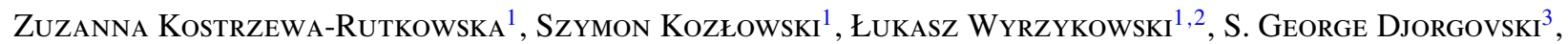 \\ Eilat Glikman ${ }^{4}$, Ashish A. Mahabal ${ }^{3}$, and Sergey Koposov ${ }^{2}$ \\ ${ }^{1}$ Warsaw University Observatory, Al. Ujazdowskie 4, 00-478 Warszawa, Poland; zkostrzewa@ astrouw.edu.pl, simkoz@astrouw.edu.pl, \\ wyrzykow@astrouw.edu.pl \\ ${ }^{2}$ Institute of Astronomy, University of Cambridge, Madingley Road, Cambridge CB3 OHA, UK \\ ${ }^{3}$ California Institute of Technology, $1200 \mathrm{E}$ California Blvd., Pasadena, CA 91125, USA \\ ${ }^{4}$ Department of Physics and Yale Center for Astronomy and Astrophysics, Yale University, P.O. Box 208121, \\ New Haven, CT 06520-8121, USA \\ Received 2013 July 23; accepted 2013 October 19; published 2013 November 13
}

\begin{abstract}
We present the discovery of a plausible super-luminous supernova (SLSN), found in the archival data of Sloan Digital Sky Survey (SDSS) Stripe 82, called PSN 000123+000504. The supernova (SN) peaked at $m_{\mathrm{g}}<19.4 \mathrm{mag}$ in the second half of 2005 September, but was missed by the real-time SN hunt. The observed part of the light curve (17 epochs) showed that the rise to the maximum took over 30 days, while the decline time lasted at least 70 days (observed frame), closely resembling other SLSNe of SN 2007bi type. The spectrum of the host galaxy reveals a redshift of $z=0.281$ and the distance modulus of $\mu=40.77$ mag. Combining this information with the SDSS photometry, we found the host galaxy to be an LMC-like irregular dwarf galaxy with an absolute magnitude of $M_{B}=-18.2 \pm 0.2 \mathrm{mag}$ and an oxygen abundance of $12+\log [\mathrm{O} / \mathrm{H}]=8.3 \pm 0.2$; hence, the $\mathrm{SN}$ peaked at $M_{\mathrm{g}}<-21.3 \mathrm{mag}$. Our SLSN follows the relation for the most energetic/super-luminous SNe exploding in low-metallicity environments, but we found no clear evidence for SLSNe to explode in low-luminosity (dwarf) galaxies only. The available information on the PSN 000123+000504 light curve suggests the magnetar-powered model as a likely scenario of this event. This SLSN is a new addition to a quickly growing family of super-luminous $\mathrm{SNe}$.
\end{abstract}

Key words: supernovae: general - supernovae: individual (PSN 000123+000504)

\section{INTRODUCTION}

There are a growing number of supernovae $(\mathrm{SNe})$ whose brightness greatly exceeds that of "classic" SNe (e.g., Quimby et al. 2011), hence not fitting into any of the standard SN classes. It is widely assumed, albeit somewhat arbitrarily, that an SN becomes a super-luminous supernova (SLSN) if its absolute magnitude exceeds $-21.0 \mathrm{mag}$ in any filter, which corresponds to the peak luminosity of $>7 \times 10^{43} \mathrm{erg} \mathrm{s}^{-1}$. During the past few years most SLSN events have been discovered by several untargeted surveys, for example, the Catalina Real-Time Transient Survey (Drake et al. 2009) and the Palomar Transient Factory (Law et al. 2009; Rau et al. 2009). It was found that these over-luminous SNe tend to prefer low-metallicity, lowmass dwarf galaxies (Prieto et al. 2008; Stanek et al. 2006; Neill et al. 2011).

The SLSNe are associated with deaths of the most massive stars, which means that they have impact on the chemical evolution and re-ionization of the universe. The SLSN explosions are probably induced by different physical mechanisms than other, more common types of $\mathrm{SNe}$, which makes these transients more interesting. However, still little is known about the nature of SLSNe, because of insufficient size and low heterogeneity of the available sample (for a recent review, see Gal-Yam 2012). The SLSN family has been divided into three classes: SLSNI (hydrogen-poor), SLSN-II (hydrogen-rich), and SLSN-R (radioactively powered). SLSN-R type seems to be the best understood one as these $\mathrm{SNe}$ are powered by large amounts of radioactive ${ }^{56} \mathrm{Ni}$ produced during the explosions of massive stars. The mechanism of the most commonly observed SLSN-II class can be explained by an explosion within a thick hydrogen envelope, but the proper energy source is still unknown.
The final group, SLSN-I explosions, is probably a result of the pair-instability mechanism, but these $\mathrm{SNe}$ are not powered by radioactivity. Recently a different statement was presented by Inserra et al. (2013). These authors claim that type SLSN-I can be classified as SLSN-Ic, because their spectral features are similar to typical Ic explosions, i.e., the blue continuum with broad absorption lines of intermediate-mass elements such as $\mathrm{C}, \mathrm{O}, \mathrm{Si}$, and $\mathrm{Mg}$, with no clear evidence of $\mathrm{H}$ and $\mathrm{He}$. Moreover, the energy deposited by newborn magnetars was favored as the power source for these events instead of the pair-instability mechanism. A semi-analytical diffusion model with energy input from the spin-down of a magnetar reproduces the extensive light-curve data well. The ejecta velocities and temperatures required by the model predictions are in a reasonable agreement with those determined from the photometric and spectroscopic observations. There are therefore some discrepancies in views on the real nature of the SLSNe, which are caused by a very small number of known examples of such explosions.

In this paper, we present the discovery of another object belonging to the class of SLSNe found in the Sloan Digital Sky Survey (SDSS) Stripe 82 multi-band data. To date there are $\sim 30$ known SLSNe; therefore, each new event observed is a valuable addition to the class. With its peak brightness of $M_{g}<-21.3$ mag (without including the $K$-correction and host galaxy extinction) and the optical light-curve shape, it closely resembles SLSNe. The subsequent sections include a detailed description of our findings.

\section{THE SUPERNOVA}

The SDSS repeatedly observed several selected areas of the sky. One of them, called Stripe 82, covering $\sim 270 \mathrm{deg}^{2}$ 

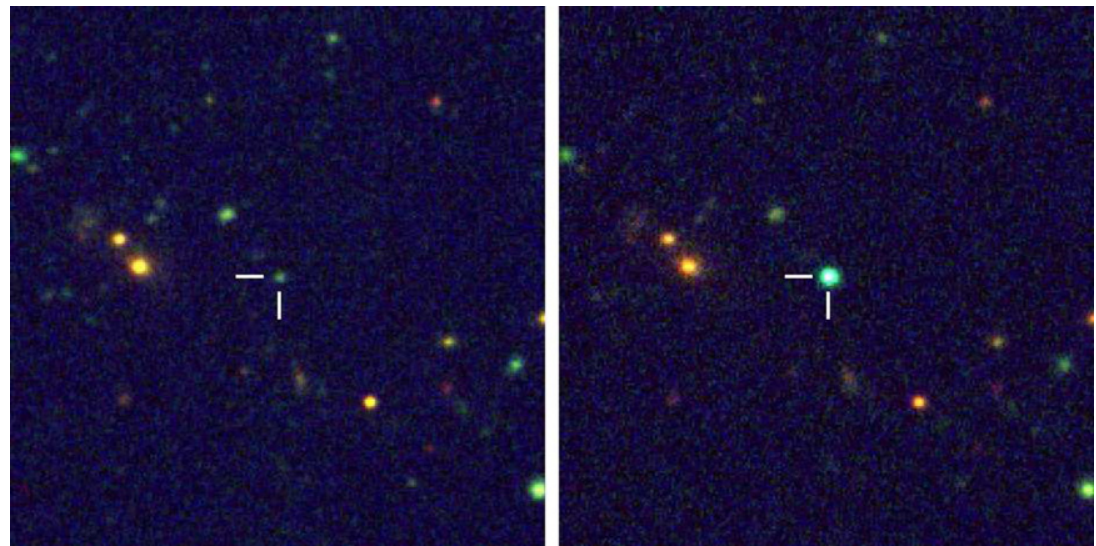

Figure 1. Finding chart for PSN 000123+000504. In the left panel, cross-hair marks the SN host galaxy, while the right panel shows the image taken near the peak of the SN explosion. The red giant branch image is a composite of $u-, r$-, and $i$-band filters and covers $1.5 \times 1$ '.5. North is up, east is to the left.

of the sky, was specifically targeted for multiple photometric observations (Ivezić et al. 2007) in the hunt for Type Ia SN explosions. There were about $500 \mathrm{SNe}$ found in near-real-time data analysis, many of them followed up spectroscopically (Frieman et al. 2008; Sako et al. 2008). The Stripe 82 covers the area of $-50^{\circ}<\alpha<59^{\circ},-1.25^{\circ}<\delta<1.25^{\circ}$. We used the catalog of Bramich et al. (2008), which provided not only the photometric and astrometric time series for almost 4 million objects, but also numerous high-level data products, useful for an effective querying of the catalog. We aimed at transients that lasted between 40 and 150 days and had mean luminosities brighter than 20.5 mag in $g$ and $u$ bands. In addition, we only looked for objects with a significant excess in blue at the peak. We found about 200 potentially interesting objects, most of them turning out to be previously found, known $\mathrm{SNe}$ (Frieman et al. 2008; Sako et al. 2008). From the remainder we selected one transient with a well-covered light curve, exhibiting long rise and long decline, as a possible SLSN. The transient was also located on top of a faint galaxy-like object (according to the SDSS Data Release 9; DR9 ${ }^{5}$ ), which ruled out the possibility of a cataclysmic variable transient. The coordinates of the $\mathrm{SN}$ are $(\alpha, \delta)=(00: 01: 23.36,+00: 05: 04.02)$, and the finding chart is presented in Figure 1. We dubbed the SN with PSN 000123+000504 "Vernal Equinox Supernova 2005" because of its position very close to the beginning of the equatorial coordinate system. The observed light curve of the $\mathrm{SN}$ is shown in Figure 2. It is also available in tabular form for the observed magnitudes (Table 1), absolute magnitudes (Table 2), and luminosity (Table 3).

It is not clear why the object was not found by a regular search for SNe during the Stripe 82 observations, which were designed for a real-time SN hunt. Most probably, the host galaxy was not recognized as a galaxy due to its compactness and blue color, suggesting a galactic progenitor of some sort of cataclysmic variable. Only after producing the final SDSS images of this region (in SDSS DR9) was the object classified as a galaxy.

While we do not have the spectrum for the SN during explosion, we can only rely on post-explosion observations. In the next section we present the evidence for the SN host galaxy redshift of $z=0.281$, which corresponds to the distance modulus of $\mu=40.77 \mathrm{mag}$ and the luminosity distance of $1428 \mathrm{Mpc}$, assuming a standard $\Lambda \mathrm{CDM}$ cosmological model

\footnotetext{
5 http://skyserver.sdss3.org/public/en/tools/chart/navi.asp
}

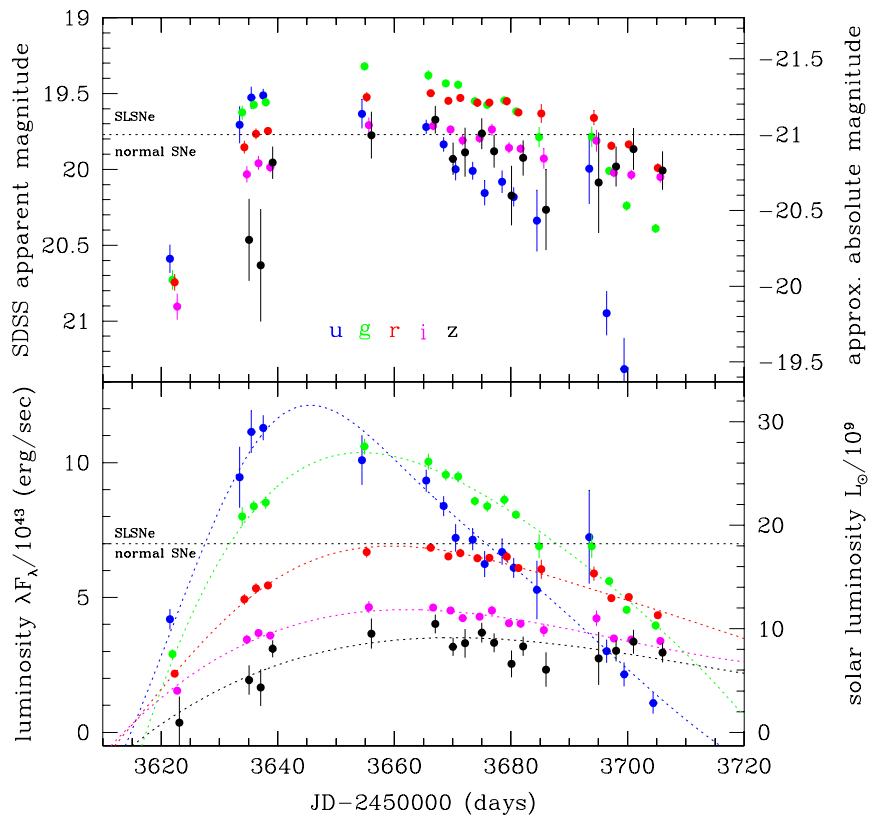

Figure 2. Light curves of PSN 000123+000504. Top panel: the SN extinctioncorrected observed magnitudes in the AB magnitude system and the approximate absolute magnitudes (host extinction and $K$-corrections not included) for five SDSS filters (marked with colors). For clarity, data points are shifted by -0.8 ( $u$ band), -0.4 ( $g$ band), +0.4 ( $i$ band), +0.8 ( $z$ band) days with respect to the observed date. The horizontal dashed line marks the division between normal SNe and SLSNe from Gal-Yam (2012). Bottom panel: the absolute magnitudes from the top panel converted to luminosity. The SN peaked above $10^{44} \mathrm{erg} \mathrm{s}^{-1}$, typical for SLSNe and atypical for normal SNe. The combined peak luminosity from five filters exceeded $3.5 \times 10^{44} \mathrm{erg} \mathrm{s}^{-1}$ (Table 3).

with $\left(H_{0}, \Omega_{M}, \Omega_{\mathrm{vac}}, \Omega_{k}\right)=\left(71 \mathrm{~km} \mathrm{~s}^{-1} \mathrm{Mpc}^{-1}, 0.27,0.73,0.0\right)$. The Milky Way (MW) extinctions in the direction of the SN are $\left(A_{u}, A_{g}, A_{r}, A_{i}, A_{z}\right)=(0.128,0.100,0.069,0.051,0.038)$ mag (Schlegel et al. 1998).

The absolute magnitude of the SN observed at magnitude $m$ is described by the equation

$$
M=m-\mu-A_{\mathrm{MW}}-A_{\mathrm{H}}(z)-K\left(z, A_{\mathrm{H}}(z)\right),
$$

where $\mu=5 \log d\left(z, \Omega_{X}\right)+25$ is the distance modulus, $d\left(z, \Omega_{X}\right)$ is the luminosity distance in Mpc, a function of redshift $z$ and cosmological model $\Omega_{X}, A_{\mathrm{MW}}$ and $A_{\mathrm{H}}(z)$ are the extinctions in our own MW and in the SN host galaxy, respectively, and $K\left(z, A_{\mathrm{H}}(z)\right)$ is the single filter $K$-correction (see, e.g., Kim et al. 1996). 
Table 1

The SN Observed Magnitudes in the AB Magnitude System

\begin{tabular}{lcccccccccc}
\hline \hline $\begin{array}{l}\mathrm{HJD}^{\prime} \\
\text { (days) }\end{array}$ & $u$ & $\sigma_{u}$ & $g$ & $\sigma_{g}$ & $r$ & $\sigma_{r}$ & $i$ & $\sigma_{i}$ & $z$ & $\sigma_{z}$ \\
\hline 3622.32727 & 20.588 & 0.093 & 20.728 & 0.065 & 20.745 & 0.055 & 20.904 & 0.086 & 22.295 & 1.424 \\
3634.27689 & 19.705 & 0.122 & 19.625 & 0.047 & 19.854 & 0.043 & 20.033 & 0.053 & 20.465 & 0.270 \\
3636.28243 & 19.527 & 0.073 & 19.575 & 0.028 & 19.766 & 0.036 & 19.960 & 0.042 & 20.632 & 0.372 \\
3638.31822 & 19.513 & 0.044 & 19.557 & 0.029 & 19.746 & 0.022 & 19.987 & 0.033 & 19.956 & 0.106 \\
3655.25412 & 19.634 & 0.095 & 19.320 & 0.028 & 19.523 & 0.032 & 19.709 & 0.051 & 19.774 & 0.154 \\
3666.24223 & 19.720 & 0.047 & 19.380 & 0.032 & 19.497 & 0.020 & 19.714 & 0.027 & 19.673 & 0.091 \\
3669.23771 & 19.835 & 0.047 & 19.433 & 0.024 & 19.549 & 0.021 & 19.737 & 0.026 & 19.930 & 0.104 \\
3671.32237 & 19.999 & 0.074 & 19.442 & 0.024 & 19.529 & 0.025 & 19.808 & 0.035 & 19.887 & 0.162 \\
3674.22166 & 20.009 & 0.058 & 19.550 & 0.020 & 19.561 & 0.024 & 19.795 & 0.031 & 19.764 & 0.102 \\
3676.31101 & 20.157 & 0.081 & 19.575 & 0.026 & 19.559 & 0.021 & 19.738 & 0.040 & 19.881 & 0.108 \\
3679.28270 & 20.081 & 0.074 & 19.545 & 0.025 & 19.551 & 0.027 & 19.858 & 0.037 & 20.172 & 0.196 \\
3681.27407 & 20.181 & 0.064 & 19.617 & 0.022 & 19.625 & 0.019 & 19.863 & 0.028 & 19.924 & 0.116 \\
3685.23284 & 20.338 & 0.200 & 19.786 & 0.067 & 19.632 & 0.063 & 19.929 & 0.066 & 20.266 & 0.265 \\
3694.24599 & 19.996 & 0.233 & 19.786 & 0.067 & 19.661 & 0.047 & 19.810 & 0.072 & 20.087 & 0.332 \\
3697.22983 & 20.948 & 0.146 & 20.011 & 0.027 & 19.844 & 0.027 & 20.022 & 0.030 & 19.981 & 0.131 \\
3700.22852 & 21.316 & 0.205 & 20.241 & 0.032 & 19.836 & 0.025 & 20.036 & 0.034 & 19.867 & 0.136 \\
3705.21473 & 22.056 & 0.333 & 20.389 & 0.030 & 19.990 & 0.030 & 20.052 & 0.034 & 20.007 & 0.127 \\
\hline
\end{tabular}

Note. $\mathrm{HJD}^{\prime}=\mathrm{HJD}-2,450,000$; the magnitudes were corrected for the Milky Way's extinction and put on the AB magnitude system.

Table 2

The SN Absolute Magnitudes in the AB Magnitude System

\begin{tabular}{lcccccccccc}
\hline \hline $\begin{array}{l}\text { HJD' } \\
\text { (days) }\end{array}$ & $u$ & $\sigma_{u}$ & $g$ & $\sigma_{g}$ & $r$ & $\sigma_{r}$ & $i$ & $\sigma_{i}$ & $z$ & $\sigma_{z}$ \\
\hline 3622.32727 & -20.182 & 0.093 & -20.042 & 0.065 & -20.025 & 0.055 & -19.866 & 0.086 & -18.475 & 1.424 \\
3634.27689 & -21.065 & 0.122 & -21.145 & 0.047 & -20.917 & 0.043 & -20.738 & 0.053 & -20.306 & 0.270 \\
3636.28243 & -21.243 & 0.073 & -21.195 & 0.028 & -21.004 & 0.036 & -20.810 & 0.042 & -20.138 & 0.372 \\
3638.31822 & -21.257 & 0.044 & -21.213 & 0.029 & -21.024 & 0.022 & -20.783 & 0.033 & -20.814 & 0.106 \\
3655.25412 & -21.136 & 0.095 & -21.450 & 0.028 & -21.247 & 0.032 & -21.061 & 0.051 & -20.996 & 0.154 \\
3666.24223 & -21.050 & 0.047 & -21.390 & 0.032 & -21.273 & 0.020 & -21.056 & 0.027 & -21.097 & 0.091 \\
3669.23771 & -20.935 & 0.047 & -21.337 & 0.024 & -21.221 & 0.021 & -21.033 & 0.026 & -20.840 & 0.104 \\
3671.32237 & -20.771 & 0.074 & -21.328 & 0.024 & -21.241 & 0.025 & -20.962 & 0.035 & -20.883 & 0.162 \\
3674.22166 & -20.761 & 0.058 & -21.220 & 0.020 & -21.209 & 0.024 & -20.975 & 0.031 & -21.006 & 0.102 \\
3676.31101 & -20.614 & 0.081 & -21.195 & 0.026 & -21.211 & 0.021 & -21.033 & 0.040 & -20.889 & 0.108 \\
3679.28270 & -20.689 & 0.074 & -21.225 & 0.025 & -21.219 & 0.027 & -20.912 & 0.037 & -20.598 & 0.196 \\
3681.27407 & -20.589 & 0.064 & -21.153 & 0.022 & -21.146 & 0.019 & -20.907 & 0.028 & -20.846 & 0.116 \\
3685.23284 & -20.432 & 0.200 & -20.984 & 0.067 & -21.138 & 0.063 & -20.841 & 0.066 & -20.504 & 0.265 \\
3694.24599 & -20.774 & 0.233 & -20.984 & 0.067 & -21.109 & 0.047 & -20.960 & 0.072 & -20.683 & 0.332 \\
3697.22983 & -19.822 & 0.146 & -20.759 & 0.027 & -20.926 & 0.027 & -20.748 & 0.030 & -20.789 & 0.131 \\
3700.22852 & -19.454 & 0.205 & -20.529 & 0.032 & -20.934 & 0.025 & -20.734 & 0.034 & -20.903 & 0.136 \\
3705.21473 & -18.714 & 0.333 & -20.381 & 0.030 & -20.780 & 0.030 & -20.718 & 0.034 & -20.763 & 0.127 \\
\hline
\end{tabular}

Note. $\mathrm{HJD}^{\prime}=\mathrm{HJD}-2,450,000$; the magnitudes were corrected for the Milky Way's extinction and put on the AB magnitude system, while using the distance modulus $\mu=40.77 \mathrm{mag}$.

There are two unknowns in Equation (1). First of all, we do not know the value of $A_{\mathrm{H}}(z)$, which is the internal host extinction at the SN location. However, because it can only make the SN brighter (the larger the value, the brighter the $\mathrm{SN}$ ), it does not affect our interpretation of the $\mathrm{SN}$ as a super-luminous one. The second unknown is the $K$-correction, as for its calculation we would need a spectrum near the peak. On the other hand, the $K$-correction at such a low redshift is not going to change the absolute magnitudes by more than a few tenths of magnitude in either direction.

We converted the absolute magnitudes to luminosity (bottom panel of Figures 2 and 3, and Table 3). Integrating the fiveband SDSS light curves over the duration of the transient, we estimated that the total emitted energy exceeded $2.1 \times 10^{51} \mathrm{erg}$. The bottom panel of Figure 2 shows the luminosity light curves. The first obvious finding is that the $u$-band (near-UV) light curve peaks first (at epoch 4 of the light curve), followed by optical light curves (at epochs 5-6), while the infrared light curves peak as the last ones, at later epochs. This obviously reflects the changes in the spectrum of the object that are characteristic for an object with high early temperatures and low temperatures at late times-clear signs of an explosion or eruption. For each epoch we constructed the five-point spectral energy distribution (SED) from the photometric data and investigated the evolution of the SED shape with time. In Figure 3, we present the SN SED at selected epochs $1,4,6$, and 16, corresponding to $-16,0,+28$, and +62 days from the $u$-band peak at JD $=2,453,638.32$ (2005 September 24). We fitted a blackbody (BB) spectrum at each epoch and obtained the best-fitting $\mathrm{BB}$ temperatures $\left(T_{\mathrm{BB}}\right)$. The BB fits to the early-time (pre-peak) SEDs returned satisfying $\chi^{2} /$ dof $<1.5$, pointing to rather featureless spectra with high temperatures $T_{\mathrm{BB}} \gtrsim 15,000 \mathrm{~K}$. The situation changes 


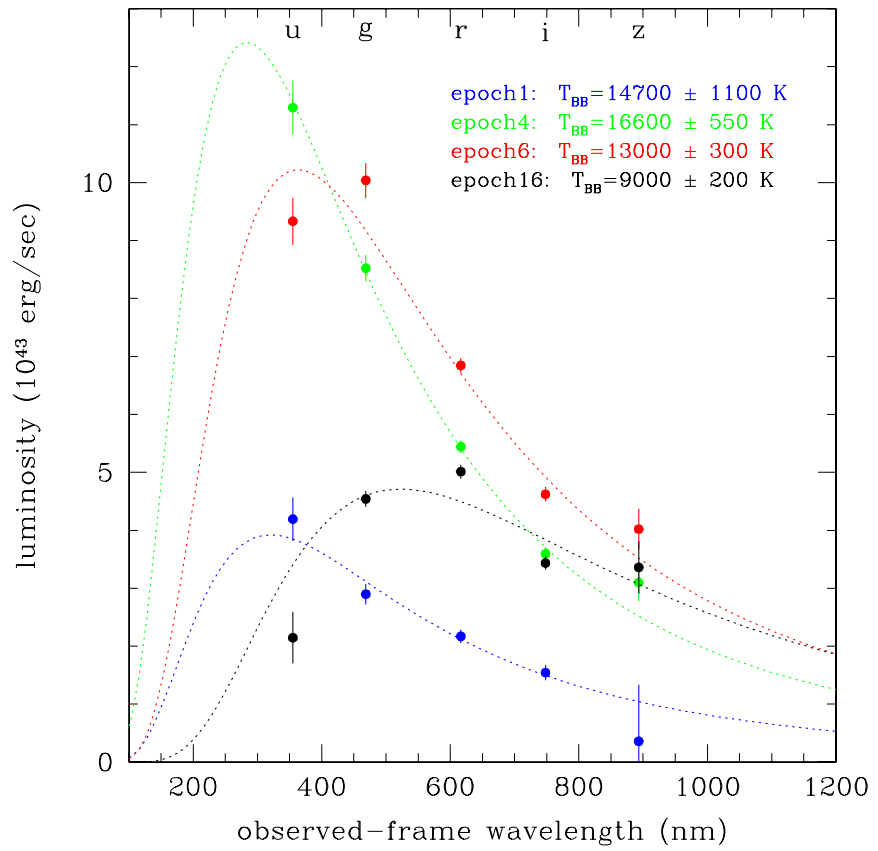

Figure 3. Blackbody at $z=0.281$ fits to the SED constructed from the photometric multi-band data for epochs 1 (blue), 4 (green), 6 (red), and 16 (black), corresponding to $-16,0,+28$, and +62 days from the $u$-band peak. While the fits to the pre-peak epochs return good blackbody matches (with $\chi^{2} /$ dof $<1.5$ ), this is not the case for late epochs. This is indicative of a significant line evolution at late epochs, characteristic of core-collapse SNe. The luminosities were corrected for Galactic extinction.

when we fit the BB spectrum to the late-time (post-peak) SEDs. The fits are statistically unacceptable with $7<\chi^{2} /$ dof $<14$. This is easily explained if we assume that our object is to find an SN. Filippenko (1997) describes a normal SN Type II-P as having a featureless blue $\mathrm{BB}$ spectrum $T_{\mathrm{BB}}>10,000 \mathrm{~K}$ at early times and a significant line evolution at late times with temperature reaching $\sim 5000 \mathrm{~K}$ a few weeks later (adiabatic expansion and cooling of the ejecta)—a picture perfectly fitting into our findings (if ignoring the high luminosity).

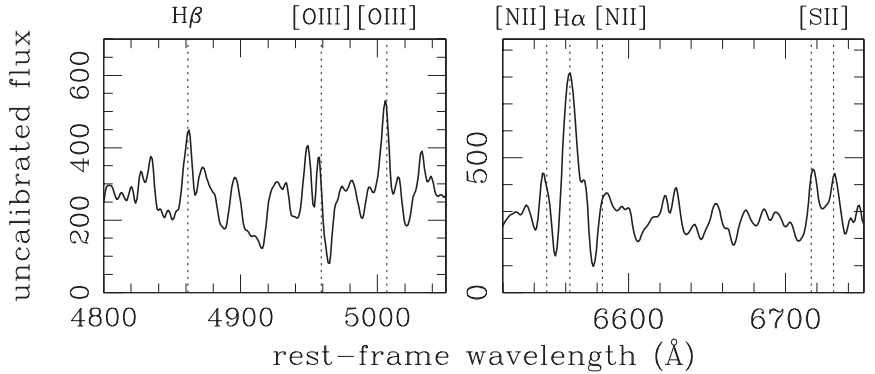

Figure 4. Selected parts of the Keck/LRIS spectrum with the major host emission lines used for redshift determination. The spectrum has a relatively low signal-to-noise ratio; nevertheless, it was sufficient to secure the redshift of $z=0.281$ and host's metallicity of $12+\log [\mathrm{O} / \mathrm{H}] \approx 8.3$. Vertical dotted lines with labels mark major emission lines.

\section{THE HOST OF PSN $000123+000504$}

The SN was found to be a transient in the Stripe 82 data, i.e., with no prior historical observations, forming a baseline. However, there is a faint galaxy-like object present in the SDSS images (Ahn et al. 2012) at the location of PSN 000123+000504. Its brightness in the SDSS DR9 is $(u, g, r, i, z)=(24.50 \pm 0.91,23.12 \pm 0.21,22.06 \pm$ $0.14,21.88 \pm 0.16,22.43 \pm 0.73) \mathrm{mag}$ in the $\mathrm{AB}$ magnitude system.

We obtained a 30 minute Keck/LRIS spectrum of the host galaxy on MJD = 56,215.353347 and 56,215.353455 (2012 October 15). While the spectrum has a rather low overall signalto-noise ratio $(\mathrm{S} / \mathrm{N})$, we were able to identify (among others) the following major emission lines: $\mathrm{H} \beta$ at $4861 \AA$, [O III] at $4959 \AA$ and $5007 \AA, \mathrm{H} \alpha$ at $6863 \AA$, and [N II] at $6884 \AA$, at a redshift $z=0.281$ (Figure 4 ).

The red part of the spectrum was then also used to estimate the host's oxygen abundance $(12+\log [\mathrm{O} / \mathrm{H}])$. We measured the emission lines $\mathrm{H} \beta$ with $\mathrm{S} / \mathrm{N}=7.4$, [O III] at $4959 \AA$ with $\mathrm{S} / \mathrm{N} \approx 0.5,[\mathrm{O}$ III] at $5007 \AA$ with $\mathrm{S} / \mathrm{N}=11.3, \mathrm{H} \alpha$ with $\mathrm{S} / \mathrm{N}=19.7$, and $[\mathrm{N}$ II] at $6884 \AA$ with $\mathrm{S} / \mathrm{N}=2.8$ to measure the $N 2$ and $O 3 N 2$ metallicity indices. Using equations from Pettini $\&$ Pagel (2004), we found $12+\log [\mathrm{O} / \mathrm{H}]=8.29$ with a formal

Table 3

Luminosity of the SN in Units of $10^{43} \mathrm{erg} \mathrm{s}^{-1}$

\begin{tabular}{|c|c|c|c|c|c|c|c|c|c|c|c|c|}
\hline $\begin{array}{l}\mathrm{HJD}^{\prime} \\
\text { (days) }\end{array}$ & $u$ & $\sigma_{u}$ & $g$ & $\sigma_{g}$ & $r$ & $\sigma_{r}$ & $i$ & $\sigma_{i}$ & $z$ & $\sigma_{z}$ & Sum & $\sigma_{\text {sum }}$ \\
\hline 3622.32727 & 4.193 & 0.376 & 2.898 & 0.180 & 2.170 & 0.114 & 1.544 & 0.128 & 0.359 & 0.974 & 11.164 & 1.073 \\
\hline 3634.27689 & 9.458 & 1.127 & 8.008 & 0.351 & 4.931 & 0.197 & 3.445 & 0.174 & 1.939 & 0.548 & 27.781 & 1.327 \\
\hline 3636.28243 & 11.140 & 0.776 & 8.385 & 0.218 & 5.344 & 0.180 & 3.684 & 0.145 & 1.661 & 0.678 & 30.214 & 1.078 \\
\hline 3638.31822 & 11.293 & 0.467 & 8.521 & 0.227 & 5.443 & 0.110 & 3.593 & 0.112 & 3.098 & 0.317 & 31.948 & 0.628 \\
\hline 3655.25412 & 10.095 & 0.922 & 10.600 & 0.274 & 6.683 & 0.199 & 4.642 & 0.224 & 3.661 & 0.559 & 35.681 & 1.152 \\
\hline 3666.24223 & 9.333 & 0.409 & 10.037 & 0.300 & 6.845 & 0.127 & 4.622 & 0.118 & 4.020 & 0.353 & 34.857 & 0.641 \\
\hline 3669.23771 & 8.389 & 0.374 & 9.554 & 0.216 & 6.528 & 0.129 & 4.522 & 0.111 & 3.171 & 0.320 & 32.164 & 0.563 \\
\hline 3671.32237 & 7.214 & 0.508 & 9.480 & 0.208 & 6.648 & 0.156 & 4.235 & 0.139 & 3.301 & 0.530 & 30.878 & 0.791 \\
\hline 3674.22166 & 7.148 & 0.393 & 8.576 & 0.161 & 6.455 & 0.144 & 4.287 & 0.125 & 3.697 & 0.363 & 30.163 & 0.590 \\
\hline 3676.31101 & 6.242 & 0.483 & 8.387 & 0.201 & 6.465 & 0.125 & 4.521 & 0.168 & 3.319 & 0.347 & 28.934 & 0.661 \\
\hline 3679.28270 & 6.690 & 0.474 & 8.622 & 0.200 & 6.515 & 0.164 & 4.046 & 0.140 & 2.539 & 0.501 & 28.412 & 0.749 \\
\hline 3681.27407 & 6.100 & 0.368 & 8.069 & 0.163 & 6.088 & 0.110 & 4.027 & 0.107 & 3.189 & 0.359 & 27.473 & 0.560 \\
\hline 3685.23284 & 5.280 & 1.070 & 6.901 & 0.440 & 6.046 & 0.360 & 3.791 & 0.238 & 2.328 & 0.642 & 24.346 & 1.391 \\
\hline 3694.24599 & 7.238 & 1.729 & 6.904 & 0.439 & 5.888 & 0.261 & 4.228 & 0.290 & 2.744 & 0.980 & 27.002 & 2.072 \\
\hline 3697.22983 & 3.011 & 0.434 & 5.612 & 0.140 & 4.974 & 0.126 & 3.480 & 0.099 & 3.028 & 0.390 & 20.105 & 0.621 \\
\hline 3700.22852 & 2.146 & 0.445 & 4.542 & 0.137 & 5.012 & 0.119 & 3.435 & 0.108 & 3.360 & 0.449 & 18.495 & 0.666 \\
\hline 3705.21473 & 1.085 & 0.389 & 3.960 & 0.111 & 4.347 & 0.123 & 3.385 & 0.106 & 2.954 & 0.366 & 15.731 & 0.569 \\
\hline
\end{tabular}

Note. HJD' = HJD -2,450,000; the luminosity is corrected for the Milky Way's extinction. 


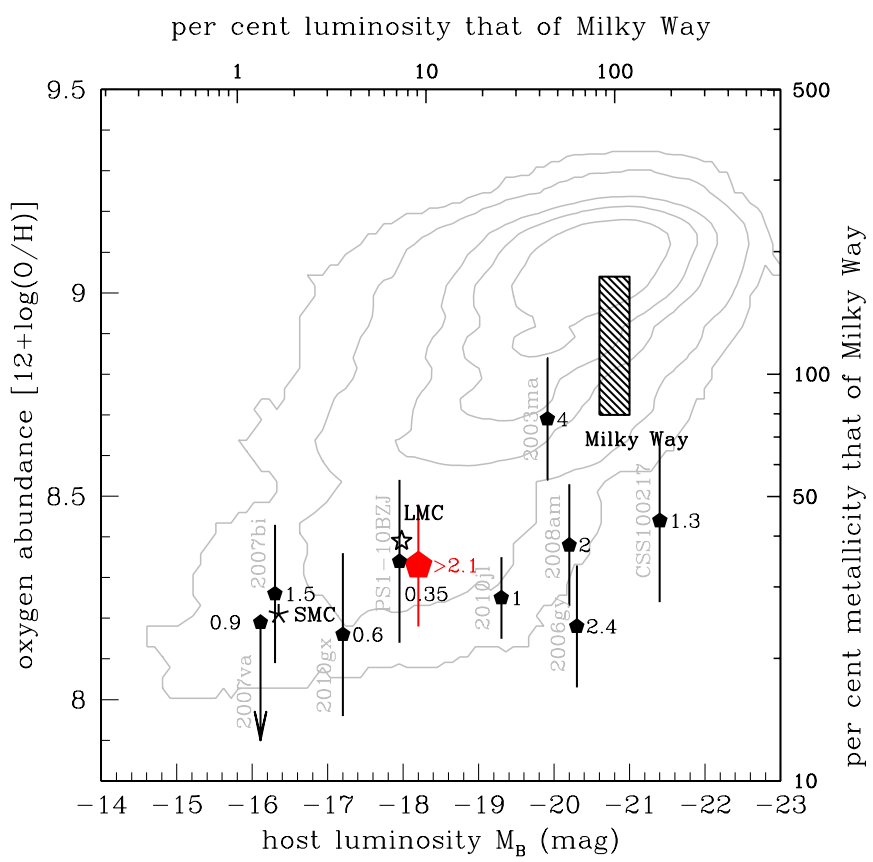

Figure 5. Metallicity-luminosity plane for 125,958 SDSS DR4 galaxies from Prieto et al. (2008) on the metallicity scale from Tremonti et al. (2004). The plane was divided into $\Delta M_{\mathrm{B}}=0.1 \mathrm{mag}$ and $\Delta(12+\log [\mathrm{O} / \mathrm{H}])=0.025 \mathrm{dex}$ bins. The contours are for 2, 10, 50, 100, and 200 galaxies per bin, smoothed with a $3 \times 3$ bin window. The MW's, LMC's, and SMC's locations are marked with a box, open star, and narrow star, respectively. Full pentagons mark nine SLSN hosts. Numbers indicate the amount of energy (in units of $10^{51} \mathrm{erg}$ ) emitted during these explosions (points taken from Kozłowski et al. 2010; Stoll et al. 2011). The PSN $000123+000504$ host galaxy is marked with a large red pentagon.

error of 0.15 for the $N 2$ index, and $12+\log [\mathrm{O} / \mathrm{H}]=8.33$ with a formal error of 0.08 for the $O 3 N 2$ index. We added in quadrature the uncertainty for the Pettini \& Pagel (2004) relations $(\sigma=$ 0.19 and 0.13 , respectively) to obtain $12+\log [\mathrm{O} / \mathrm{H}]=8.33 \pm$ 0.15 for the $O 3 N 2$ index and $12+\log [\mathrm{O} / \mathrm{H}]=8.29 \pm 0.24$ for the $N 2$ index (Figure 5). Both values are consistent with each other and indicate that the SN host galaxy has $30 \%$ of MW's metallicity (adopting 8.8 from Delahaye et al. 2010).

After converting the host magnitudes to luminosity, we fit the galaxy/active galactic nucleus models of Assef et al. (2010) to the five photometric data points. The only well-fitting spectrum is that of an irregular galaxy. This supports our claim that the host galaxy is a dwarf, irregular, metal-poor galaxy. The absolute brightness of the galaxy in $B$ was obtained as $M_{\mathrm{B}}=-18.2 \pm 0.2 \mathrm{mag}$, and the galaxy was placed on the absolute magnitude-metallicity plane, as shown in Figure 5.

\section{DISCUSSION}

In this paper, we presented the discovery of an overlooked SN in the SDSS Stripe 82 archival data, which we classified as an SLSN based on its peak optical magnitude brighter than -21 . The $\mathrm{SN}$ was found in a search for long transient events with a significant blue excess at peak in a variability database for SDSS Stripe 82. While we do not have the spectrum for the $\mathrm{SN}$ during explosion, we present the evidence for the $\mathrm{SN}$ host galaxy redshift of $z=0.281$ using the Keck/LRIS spectrum. We also constructed the five-point SED from the photometric data and investigated its evolution as a function of time. We fitted the BB spectrum at each epoch and obtained a best-fitting $\mathrm{BB}$ temperature, greater than $15,000 \mathrm{~K}$, in early-time SEDs. The

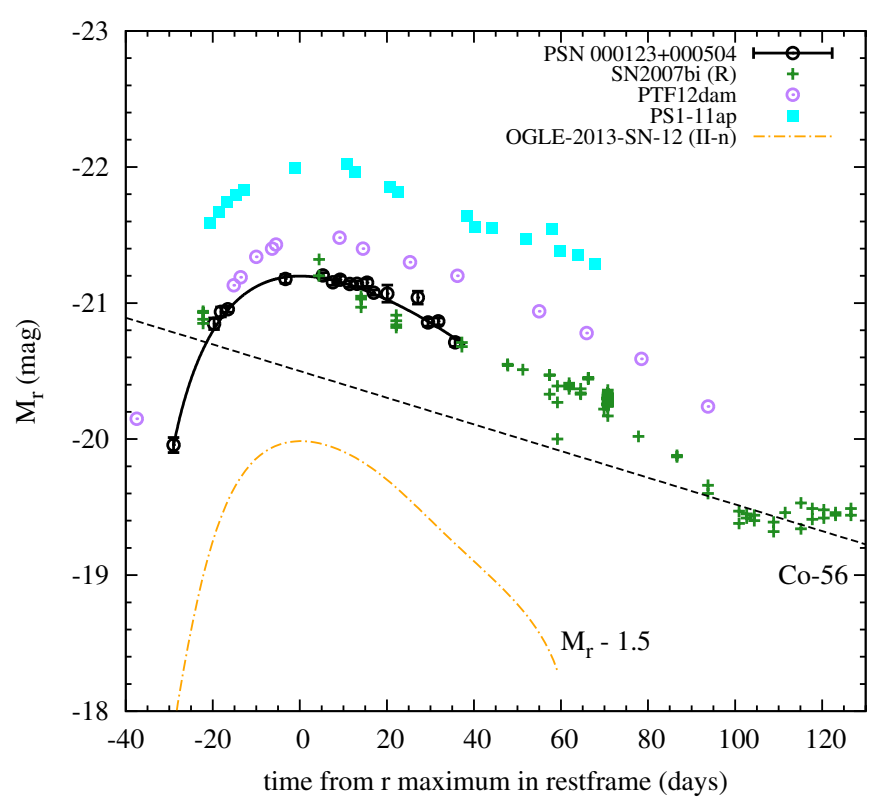

Figure 6. Absolute $r$-band light curve of PSN 000123+000504 ("Vernal Equinox Supernova 2005," black points) and other super-luminous events: PTF12dam (Nicholl et al. 2013), PS1-11 ap (McCrum et al. 2013), and SN 2007bi (Gal-Yam et al. 2009). The light curve of SN 2007bi is shown in the $R$ band. Also a Type II-n SN from the OGLE database is shown (OGLE-2013-SN-12; EliasRosa et al. 2013). The solid black line is the spline fit to the SN data. The data for each SN have been corrected for time dilation and distance modulus.

temperature evolution with a decline in late epochs to $\sim 5000 \mathrm{~K}$ also constitutes a strong piece of evidence in favor of classifying this object as an SLSN (Miller et al. 2009; Modjaz et al. 2009).

In Figure 6, we present the $r$-band light curves of our SN and a few events classified as SLSNe: SN 2007bi (Gal-Yam et al. 2009), PTF12dam (Nicholl et al. 2013), and PS1-11 ap (McCrum et al. 2013). For comparison we also show the light curve of a likely Type II-n SN from the OGLE database (Elias-Rosa et al. 2013; Wyrzykowski et al. 2012; see also Kozłowski et al. 2013 for OGLE SN overview), which is a few magnitudes fainter, but presents a similar shape of the light curve. Overall, the shape of the light curve of PSN 000123+000504 resembles very much that of objects found previously and classified as SLSNe. The rise of the light curve is very slow-it lasts about 30 days in the rest frame from the faintest detected data point to the maximum. Then, the decline is also very slow, in rough agreement with a radioactive decay of Co-56, at least in its early post-peak phase. Such behavior was in the past associated with SNe of SLSN-R type (Gal-Yam 2012); however, the suggested pair-instability origin of those SLSNe was recently questioned by Inserra et al. (2013) and Nicholl et al. (2013). They suggest that SNe similar to SN 2007bi are just a subtype of SLSN-I and are well described by a magnetar scenario. The two objects, PTF12dam and PS1-11ap, spectroscopically resemble other SLSNe, which are not radioactively driven and can be successfully modeled with magnetar heating. This makes it unnecessary to apply the pair-instability mechanism for slowly evolving and bright $\mathrm{SNe}$ (like PTF12dam, PS1-11ap, and, by implication, SN 2007bi). Nicholl et al. (2013) suggest a magnetar-powered model that can properly explain all of the data.

There is growing evidence for a correlation of the most energetic explosions preferring to occur in the low-metallicity $\left(<50 \%\right.$ that of MW) dwarf $\left(L / L_{\star}<0.1\right)$ galaxies (Stanek et al. 2006; Kozłowski et al. 2010; Stoll et al. 2011; Gal-Yam 2012). While we find the former to be true for SLSNe (Figure 5), we are 
unable to confirm the latter part of the statement. In Figure 5, we see that luminous $\mathrm{SNe}$ occupy all types of host galaxies with $-16<M_{\mathrm{B}}<-21.5 \mathrm{mag}$ (galaxies with $1 \%-200 \% \mathrm{MW}$ luminosity). Hence, there is no clear evidence for SLSNe to prefer dwarf galaxies, but this claim is based on a statistically small sample of 10 hosts.

We examined the properties of the faint host galaxy. With an oxygen abundance $(12+\log [\mathrm{O} / \mathrm{H}]) \approx 8.30$, the host galaxy of PSN $000123+000504$ has $30 \%$ of MW's metallicity and an absolute magnitude comparable to that of the Large Magellanic Cloud (LMC). The place of the SN host galaxy on the absolute magnitude-metallicity plane (Figure 5) is among other hosts with detected SLSNe. The presence of SLSNe usually indicates that the host is undergoing an episode of active star formation. The extreme luminosity of SLSNe is the key for their discovery over a range of redshifts, which helps the investigation of star formation in galaxies at high redshifts. Furthermore, the plausible high masses of their progenitors (in excess of $150 M_{\odot}$ ) present an opportunity to model the initial mass function in, e.g., low-mass galaxies. The low host masses of observed SLSNe would indicate that these objects were produced at the early stages of galactic evolution, which creates a modeling problem to obtain progenitors in such galaxies with consistent metallicity (see Young et al. 2010 for a comparison).

Based on our single detection, we can roughly estimate the SLSN rate from the SDSS data. The Stripe 82 survey covers about 270 square degrees, which corresponds to a volume of $\sim 0.28 \mathrm{Gpc}^{3}$ within redshift $z<0.4$, a distance to which we are able to detect our SLSN. The brightest part of the SN light curve in range from -20 to -21.5 mag can be seen during about 100 days (in $g$ band with a detection limit of 21.5 mag). According to these assumptions, during the $8 \mathrm{yr}$ survey only one SLSN has been detected. There is one more SLSN found in the SDSS, but it is too faint for our cutoffs (Leloudas et al. 2012). Hence, we find that the SLSN rate is of order of $-9<\log \left(\mathrm{SLSN} \mathrm{Mpc}^{-3} \mathrm{yr}^{-1}\right)<-8$ (with the assumption of $10 \%$ efficiency in SN detection for Stripe 82 data, which is low mostly due to non-uniform SDSS cadence). This result is consistent with the rates presented previously by Gal-Yam (2012).

\section{SUMMARY}

We presented a new example of the SLSN class, which was found in the archival data of the SDSS Stripe 82. The redshift of the host, $z=0.281$, determined with the Keck/ LRIS spectrum, indicates that the brightness of this $\mathrm{SN}$ in the maximum exceeded $-21 \mathrm{mag}$. The light-curve resemblance to SN 2007bi and other similar SLSNe discovered so far indicates that PSN 000123+000504 may belong to the SLSN-R class (Gal-Yam et al. 2009; Young et al. 2010) or to magnetars
(Inserra et al. 2013; Nicholl et al. 2013). This study also shows the potential of archival multi-color time-series databases for successful detection of interesting and rare cases of transients.

Based in part on the data obtained at the W. M. Keck Observatory, which is operated as a scientific partnership among the California Institute of Technology, the University of California, and the National Aeronautics and Space Administration. The Observatory was made possible by the generous financial support of the W. M. Keck Foundation.

Z.K.R. is supported from grant 2011/01/N/ST9/03069 by the Polish National Science Centre. S.K. is supported from (FP7/2007-2013)/ERC grant agreement No. 246678 awarded by the European Research Council under the European Community's Seventh Framework Programme to the OGLE project. Ł.W. acknowledges support from the "Ideas Plus" grant No. IdP2012 000162 by the Polish Ministry of Science and Higher Education. S.G.D. and A.A.M. were supported in part by the NSF grant AST-0909182.

\section{REFERENCES}

Ahn, C. P., Alexandroff, R., Allende Prieto, C., et al. 2012, ApJS, 203, 21 Assef, R. J., Kochanek, C. S., Brodwin, M., et al. 2010, ApJ, 713, 970 Bramich, D. M., Vidrih, S., Wyrzykowski, L., et al. 2008, MNRAS, 386, 887 Delahaye, F., Pinsonneault, M. H., Pinsonneault, L., \& Zeippen, C. J. 2010, arXiv: 1005.0423

Drake, A. J., Djorgovski, S. G., Mahabal, A., et al. 2009, ApJ, 696, 870

Elias-Rosa, N., Garoffolo, A. M., Benitez-Herrera, S., et al. 2013, ATel, 4774, 1

Filippenko, A. V. 1997, ARA\&A, 35, 309

Frieman, J. A., Bassett, B., Becker, A., et al. 2008, AJ, 135, 338 Gal-Yam, A. 2012, Sci, 337, 927

Gal-Yam, A., Mazzali, P., Ofek, E. O., et al. 2009, Natur, 462, 624 Inserra, C., Smartt, S. J., Jerkstrand, A., et al. 2013, ApJ, 770, 128 Ivezić, Ž., Smith, J. A., Miknaitis, G., et al. 2007, AJ, 134, 973 Kim, A., Goobar, A., \& Perlmutter, S. 1996, PASP, 108, 190

Kozłowski, S., Kochanek, C. S., Stern, D., et al. 2010, ApJ, 722, 1624 Kozłowski, S., Udalski, A., Wyrzykowski, Ł., et al. 2013, AcA, 63, 1 Law, N. M., Kulkarni, S. R., Dekany, R. G., et al. 2009, PASP, 121, 1395 Leloudas, G., Chatzopoulos, E., Dilday, B., et al. 2012, A\&A, 541, A129 McCrum, M., et al. 2013, arXiv:1310.4417

Miller, A. A., Chornock, R., Perley, D. A., et al. 2009, ApJ, 690, 1303 Modjaz, M., Li, W., Butler, N., et al. 2009, ApJ, 702, 226

Neill, J. D., Sullivan, M., Gal-Yam, A., et al. 2011, ApJ, 727, 15

Nicholl, M., Smartt, S. J., Jerkstrand, A., et al. 2013, Natur, 502, 346

Pettini, M., \& Pagel, B. E. J. 2004, MNRAS, 348, L59

Prieto, J. L., Stanek, K. Z., \& Beacom, J. F. 2008, ApJ, 673, 999

Quimby, R. M., Kulkarni, S. R., Kasliwal, M. M., et al. 2011, Natur, 474, 487

Rau, A., Kulkarni, S. R., Law, N. M., et al. 2009, PASP, 121, 1334

Sako, M., Bassett, B., Becker, A., et al. 2008, AJ, 135, 248

Schlegel, D. J., Finkbeiner, D. P., \& Davis, M. 1998, ApJ, 500, 525

Stanek, K. Z., Gnedin, O. Y., Beacom, J. F., et al. 2006, AcA, 56, 333

Stoll, R., Prieto, J. L., Stanek, K. Z., et al. 2011, ApJ, 730, 34

Tremonti, C. A., Heckman, T. M., Kauffmann, G., et al. 2004, ApJ, 613, 898

Wyrzykowski, L., Udalski, A., \& Kozlowski, S. 2012, ATel, 4495, 1

Young, D. R., Smartt, S. J., Valenti, S., et al. 2010, A\&A, 512, A70 